\title{
EDITORIAL
}

\section{The Emerging Need for Education of the Primary Care Physician in Clinical Cancer Prevention and Survivorship Care}

\section{Lonzetta L. Newman and Elise D. Cook}

Department of Clinical Cancer Prevention, Division of Cancer Prevention and Population Sciences, The University of Texas M.D. Anderson Cancer Center, Houston, Texas, USA

\begin{abstract}
There is a growing need to expand the education of the primary care physician to include cancer prevention and survivorship care. US cancer survivors are steadily on the rise, while oncologists in training and currently in practice are declining. This perspective discusses why implementation of an educational program which focuses on the primary care physician is vital to fill this gap.
\end{abstract}

\section{Keywords}

cancer prevention; medical education; primary care physician

\section{Introduction}

With continual advances in technology, pharmaceuticals, and medical techniques, the number of cancer survivors in the United States has steadily increased and that trend is likely to continue. The estimated number of cancer survivors in 2016 was 15.5 million, as reported in the Cancer Treatment and Survivorship Statistics, 2016, and that number has been projected to increase to 20.3 million within the next 10 years. ${ }^{1}$ This growing population of cancer survivors requires preventive care and personalized treatment that fully utilize our advancing knowledge. Because of the increasing shortage of oncologists, primary care physicians will have to fill the gap and provide cancer survivorship care in addition to the care they provide for other chronic diseases.

According to the physician workforce report issued by Information Handling Services Inc. to the American Association of Medical Colleges in April 2016, by 2025, the estimated shortage of physicians in all specialties including medical oncologists and surgical subspecialties will range from 61,700 to $94,700 .^{2}$ This shortage of physicians is well recognized. ${ }^{3}$ Also, the estimated number of primary care physicians in the United States as of 2014 per the Federation of State Medical Boards was 916,264, ${ }^{4}$ an inadequate number; as, there are physician-to-patient ratios in some inner-city locales similar to those of underdeveloped countries.

This workforce shortage could be mitigated in part by education of primary care physicians. An example of the knowledge gap involves the dissemination and implementation of the results from the Breast Cancer Prevention Trial published in 1998. This trial showed that tamoxifen use in women greater than the age of 60 , women with an elevated risk of breast cancer per the Gail Model, and women with lobular carcinoma in situ and atypical hyperplasia reduces the incidence of estrogen receptor-positive breast carcinomas by approximately fifty percent. ${ }^{5}$ Yet 19 years later, knowledge of such important findings is still lacking in those trained in traditional primary care medicine.

Another example is the Study of Tamoxifen and Raloxifene trial. These participants were postmenopausal women of at least 35 years of age who had an elevated risk of breast cancer per the Gail Model. The results revealed raloxifene is as effective as tamoxifen in reducing the risk of 
invasive breast cancer and has a lower risk of thromboembolic events and cataracts and no risk of endometrial carcinoma, but a nonstatistically significant higher risk of noninvasive breast cancer. ${ }^{6}$

These gaps in the implementation of advances in cancer care must be filled. Primary care physicians must be educated in cancer prevention and in the management of cancer as a chronic condition, all through personalized plans of care.

\section{Perspective from a Major Cancer Center}

The University of Texas M.D. Anderson Cancer Center, an $\mathrm{NCl}$ designated Comprehensive Care Center, has top-rated clinical care and research provided by worldrenowned faculty and staff who deliver cutting-edge cancer care through the collaborative efforts of its multidisciplinary teams structured by disease-based centers.

The Cancer Prevention Center is one of these centers, a cornerstone which provides cancer prevention, early detection, and survivorship care. Its services include:

- Assessment of individual risk factors for cancer, including body mass index, environmental and in utereo exposures, extended family history, and chemical exposures;

- Risk Calculation of breast cancer risk in particular to assess screening options and provide a catalyst for discussion and initiation of preventive therapies;

- The latest cancer screening techniques, including adjunctive screening modalities for highrisk individuals;

- Prevention medications for individuals at high risk of breast carcinoma, including selective estrogen receptor modulators and aromatase inhibitors; and

- Cancer survivorship care for breast, colon, rectal, and anal cancers.

To impart skills in these areas to primary care professionals, institutes like ours must train practicing clinicians and trainees through clinical rotations of clinical cancer prevention and survivorship care. M.D. Anderson provides such experiences in cancer prevention and survivorship care to trainees rotating in the Cancer Prevention Center, including residents in internal medicine, family medicine, and preventive medicine; advanced practice nursing students and fellows; medical students; fellows in breast diagnostic imaging, medical oncology, surgical oncology, and clinical pathology; and international healthcare professionals in observorships. Such training has been beneficial in assisting those to incorporate prevention in their clinical practices.

In addition, a unique partnership has recently developed between Baylor College of Medicine and The Division of Internal Medicine at MD Anderson. This partnership affords opportunities to train a special group of internal medicine residents to become experts in the continuum of cancer care. It represents the first of such alliances and will specifically train a group of primary care physicians in the prevention of cancer, short-term and longterm complications of cancer, survivorship, and special social needs of this growing patient population. This partnership addresses the emerging need for the integration of primary care clinician into the continuum of cancer care. $^{7}$

Internal medicine residents who elect to participate in this special Baylor/MDAnderson Cancer Center track have a dedicated 13-month pathway within their residency that entails clinical rotations in cancer prevention and in other subspecialties of internal medicine, including nephrology, pulmonary medicine, and cardiology. These rotations specifically emphasize the direct infiltrative path of a particular cancer, its treatment complications, and the adverse effects of certain treatment modalities. This educational opportunity supports training by non-oncologic experts in treating the medical complications of cancer.

In addition, for many patients, cancer has become a chronic disease and most chronic diseases are typically managed in an outpatient setting by primary care physicians. Laboratory values obtained at specific intervals, evaluations of designated target organ damage, and specific follow-up regimens are all used as modules of reimbursement in the business of medicine by insurance companies per adopted national guidelines. Cancer Survivorship Care plans directed by national guidelines should serve as the same in this unique population of patients. 
These elements of outpatient care are routine for other chronic diseases and should be available for cancer survivorship care in the future. The American Society of Clinical Oncology (ASCO) and other national cancer organizations are working on legislation to obtain payment for completion of the Survivorship Care Plan. ASCO recommends that physicians verify which services are covered with the patient's insurance company.

Facilitating the acquisition of knowledge in cancer prevention through education efforts like those described here will help the next generation of health care professionals transcend our current level of prevention and survivorship care.

Strategies for the prevention of primary cancers, early detection of cancer, management of secondary cancers related to the primary cancer, tailoring of therapies to specific pathologic conditions caused by cancer therapies, and other components of survivorship care represent important links in the spectrum of cancer care. Given the shortage of oncologists and physicians in the United States and the increasingly chronic nature of cancer survivorship care, clinical cancer prevention and survivorship care should be part of the mandatory training of primary care physicians worldwide.

The authors wish to acknowledge the Department of Scientific Publications at The University of Texas MD Anderson Cancer Center for their assistance in submission of this original work. MD Anderson Cancer Center is supported in part by the National Institutes of Health through Cancer Center Support Grant P30CA016672.

\section{References}

1. Miller K, Siegel R, Lin C, et al. Cancer Treatment and Survivorship Statistics. CA Cancer J Clin. 2016; 66(4): 271-289.

2. Association of American Medical Colleges. 2016 Update: The Complexities of Physician Supply and Demand: Projections from 2014 to 2025. Final Report submitted by IHS Inc., 2016, April 5.

3. Chang S and Collie C. The Future of Cancer Prevention: will our workforce be ready? Cancer Epidemiol Biomarkers Prev. 2009; Sep; 18(9): 2348-2351.

4. Young A, Chaudhry H, Pei X, Halbesleben K, Polk D, Dugan MA. Census of actively licensed physicians in the United States, 2014. J Med Reg. 2013; 101(2): 8-23.

5. Fisher B, Costantino J, Wickerham D, et al. Tamoxifen for prevention of breast cancer: Report of the National Surgical Adjuvant Breast and Bowel Project P-1 Study. J Natl Cancer Inst. 1998; 90 (18):13711388.

6. Vogel V, Costantino J, Wickerham L, et al. Effects of Tamoxifen versus Raloxifene on the Risk of Developing Invasive Breast Cancer and Other Disease Outcomes. The NSABP Study of Tamoxifen and Raloxifene (STAR) P-2 Trial. JAMA. 2006; 295(23): 2727-2741.

7. Lin J, Goel M, Overholser O, Nekhlydov L. General Internists and Cancer Care across the Continuum. Society of General Internal Medicine Forum. 2016; 39(9): 7,13. 